\title{
Oleuropein and Related Compounds Reduce Atherosclerosis
}

\author{
Paul L. Huang*, Philip L. Huang and Sylvia Lee-Huang \\ Cardiology Division, Department of Medicine, Massachusetts General Hospital and Harvard Medical School, and \\ Biochemistry Department, New York University School of Medicine, USA
}

\begin{abstract}
Diabetes, obesity, and the metabolic syndrome all increase the risk for cardiovascular disease. Epidemiologic studies have demonstrated that a Mediterranean diet rich in olive oil is associated with decrease in risk for cardiovascular disease, obesity, and diabetes. Although some of the protection may be from the unsaturated fatty acid components of such a diet, additional small molecules found in olive oil and olive plants may confer protection, including the polyphenol oleuropein and hydroxytyrosol. We report here studies in mice that document the bioavailability of these molecules when administered orally. Using the apoE knockout mouse model fed a high fat diet, we assessed the effects of orally administered oleuropein on diet-induced atherosclerosis. Oleuropein significantly reduced the extent of atherosclerosis found in the aorta of apoE knockout mice. Molecular modeling studies indicate that oleuropein binds to the peroxisome proliferator activating receptors (PPAR) $\alpha, \gamma$, and $\delta$, but it does so with distinct conformations that differ from the way that PPAR $\alpha-, \gamma$-, and $\delta$-specific agonists bind to their active sites. The manner with which oleuropein binds uniquely to the various PPARs offers a possible explanation for its effects on metabolism and cardiovascular disease.
\end{abstract}

Keywords: Olive, oleuropein, hydroxytyrosol, metabolic syndrome, atherosclerosis, Mediterranean diet, apoE knockout, LCMS, molecular modeling, PPAR.

\section{INTRODUCTION}

\section{Obesity, Diabetes, and Metabolic Syndrome}

Obesity increases the risk for cardiovascular disease, including hypertension, coronary artery disease, and stroke. The risk of fatal and nonfatal myocardial infarction increases with obesity. Using the body mass index (BMI) as a measure of obesity, the relative risk for coronary artery disease doubles for BMI between 25 and 29, and triples for BMI over 29, as compared with BMI less than 21 [1]. In a separate study, coronary events increase by $10 \%$ for each increase in BMI over 22 [2]. Thus, obesity by itself increases risk for cardiovascular disease.

Obesity is often linked to diabetes, and both are characterized by insulin resistance. In addition, hypertension and atherogenic dyslipidemia are often associated with obesity, leading to the concept of the metabolic syndrome. There are several definitions of the metabolic syndrome but most include the features of abdominal obesity, hypertension, dyslipidemia (including both hypertriglyceridemia and low HDL cholesterol), and hyperglycemia [3]. In addition, inflammation and hypercoagulability are thought to be involved in the pathophysiology. The underlying root causes of metabolic syndrome are thought to be primary abnormalities in adipocyte metabolism and dyslipidemia, that then lead to defective insulin sensitivity, vascular dysfunction, and inflammatory and thrombotic tendencies [4].

\footnotetext{
*Address correspondence to this author at the Cardiology Division, Department of Medicine, Massachusetts General Hospital and Harvard Medical School, and Biochemistry Department, New York University School of Medicine, USA; Tel: 001-617-724-9849; Fax: 001-617-726-5806; E-mail: HUANGP@HELIX.MGH.HARVARD.EDU
}

\section{Signaling in Fat Cells}

In addition to serving as a reservoir for the storage and release of energy, adipose tissue also responds to circulating signaling molecules, including insulin, glucagon, catecholamines, and cytokines such as the interleukins and TNF- $\alpha$. Adipose tissue also releases signaling molecules, including proteins involved in lipid metabolism, cytokines, adipokines (leptin, adiponectin, resistin), and the peroxisomeproliferator activated receptors PPAR $\alpha, \operatorname{PPAR} \gamma$, and PPAR $\delta$. These adipocyte-derived molecules are key signals that are involved in mediating the vascular abnormalities seen in obesity, metabolic syndrome, and diabetes.

The PPARs are members of a nuclear receptor superfamily that includes the steroid, thyroid, and vitamin D receptors. PPARs regulate gene expression in response to small lipid molecules that can be present in the diet, or from metabolic derivatives in the body. Thus, they serve as fat sensors, which when activated, can markedly redirect metabolism. The three PPAR family members have distinct functions and tissue distributions [5].

- $\quad$ PPAR $\alpha$ is expressed predominantly in the liver and, to a lesser extent, in cardiac and skeletal muscle. PPAR $\alpha$ plays a crucial role in fatty acid oxidation in response to fasting, providing ketone bodies that serve as an energy source for peripheral tissues. PPAR $\alpha$ agonists include the fibrates, which are used clinically to treat hypertriglyceridemia.

- $\quad$ PPAR $\gamma$ is essential for fat cell differentiation. It acts as a fatty acid sensor that regulates whole-body glucose homeostasis, and activates genes involved in lipogenesis and lipid storage. It also modulates adipokine expression, increasing production of adiponectin, while it blocks expression of TNF- $\alpha$ and 
resistin. The net result of these changes increases insulin sensitivity. PPAR $\gamma$ is the molecular target for the thiazolidinedione class of drugs clinically used to treat patients with diabetes.

- $\quad \operatorname{PPAR} \delta$, also known as PPAR $\beta$, is expressed ubiquitously, and regulates expression of genes involved in fatty acid catabolism and adaptive thermogenesis.

PPARs modulate vascular effects by affecting insulin resistance, and by expression of adipokines. In addition, PPARs may have direct effects on gene transcription in vascular tissues and endothelial cells.

\section{BIOLOGICAL ACTIVITIES OF PHYTOCHEMICALS FOUND IN OLIVE PLANTS}

\section{Effects of the Mediterranean Diet}

Population-based studies demonstrate that a Mediterranean diet, which is enriched in olive oil, is associated with protection against atherosclerosis and cancer [6]. The Mediterranean diet is based on the traditional dietary patterns of southern Italy, Crete, and coastal Greece. It consists of abundant plant foods, whole grains, and fresh fruit. Olive oil is the principal source of fat. The diet includes moderate consumption of fish and poultry, and limited red meat and eggs. However, the precise components of such a diet responsible for protection are not known, nor their molecular mechanisms of action.

\section{Lipid Components}

Olive oil contains monounsaturated fats, notably oleic acid [6]. Monounsaturated fats are associated with decreased LDL cholesterol, increased HDL cholesterol, and decreased incidence of cardiovascular disease. Fig. (1) shows the chemical structure of oleic acid.

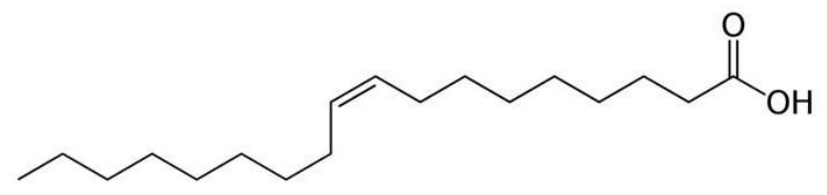

Fig. (1). Chemical structure of oleic acid.

\section{Polyphenols}

In addition to the lipid components of olive oil, phytochemicals found in olives have biological activities that may contribute to protection against atherosclerosis mediated by non-lipid components of olive oil [7-9]. One such phytochemical is the polyphenol oleuropein. The pharmacologic properties of oleuropein have been reviewed recently [10]. Oleuropein is a polar glycoside that includes a glucose molecule. Its structure is shown in Fig. (2). The glucose moiety is circled in red, and the aglycone is circled in blue.

Fig. (3) shows the metabolism of oleuropein. Enyzmatic digestion by $\beta$-glucosidase results in cleavage of the glucose moiety (circled in red) from the non-polar oleuropein aglycone. Hydrolysis of oleuropein along the blue dotted lines yields hydroxytyrosol and elenolic acid.

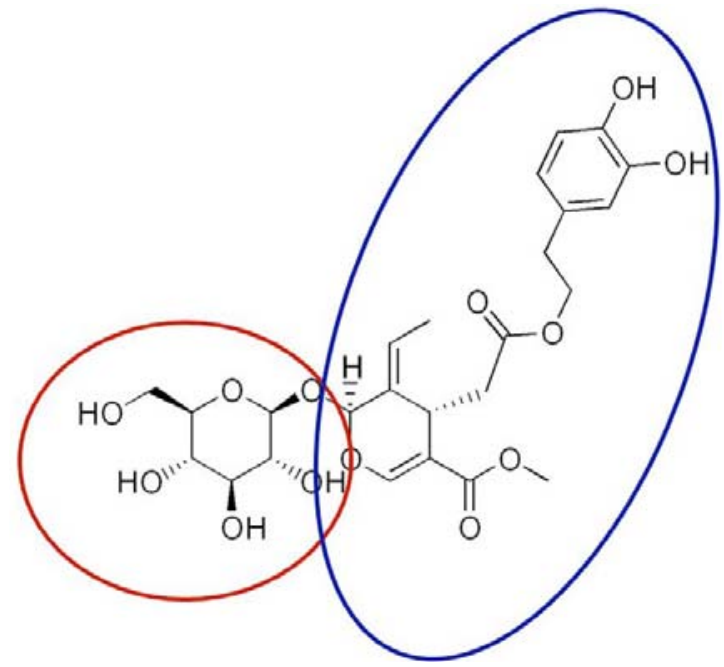

Fig. (2). Chemical structure of oleuropein, showing glucose (red) and oleuropein aglycone (blue).

In cell culture, these compounds have been shown to possess antioxidant activity [11]. They also increase the amount of bioavailable NO generated by endothelial cells [12]. However, the direct significance of these effects to atherosclerosis has not been shown in vivo. These compounds also have immunomodulatory activities $[13,14]$.

\section{IN VIVO STUDIES OF OLEUROPEIN}

\section{Bioavailability Studies of Oleuropein In vivo}

We used liquid chromatography (LC) coupled with mass spectrometry (MS) for sensitive and specific identification and quantitation of oleuropein. Fig. (4A) shows the LC profile of oleuropein standard. The three profiles are absorption at $230 \mathrm{~nm}$ (top), $280 \mathrm{~nm}$ (middle), and total ionic current (bottom). Fig. (4B) shows the MS of the oleuropein standard, with the major peak at molecular mass 539 corresponding to intact oleuropein.

Using LC-MS, we determined that chronic administration of olive leaf extract to rats and mice results in absorption of biologically active compounds in vivo.

\section{Effects of Oleuropein on Diet-Induced Atherosclerosis in apoE Knockout Mice}

The apoE knockout mouse is the most frequently used rodent model of hypercholesterolemia. ApoE knockout mice develop atherosclerosis with vascular distribution and histopathologic features similar to those seen in human cardiovascular disease $[15,16]$. When fed a Western diet containing $42 \%$ of calories from fat, apoE knockout mice show accelerated and reproducible formation of atherosclerotic lesions. Histologically, these lesions show fatty streaks, monocyte adherence, and foam cells similar to those seen in man. The degree of atherosclerosis can be quantitated by en face staining with Oil Red O or by sections through the aortic root cusps.

To determine the effects of oleuropein on atherosclerosis, we administered oleuropein to apoE knockout mice in their drinking water at concentrations of $0.25 \mathrm{mg} / \mathrm{ml}, 2.5 \mathrm{mg} / \mathrm{ml}$, and $25 \mathrm{mg} / \mathrm{ml}$ for 4 weeks. Even at the lowest dose, there was a significant reduction in the development of 


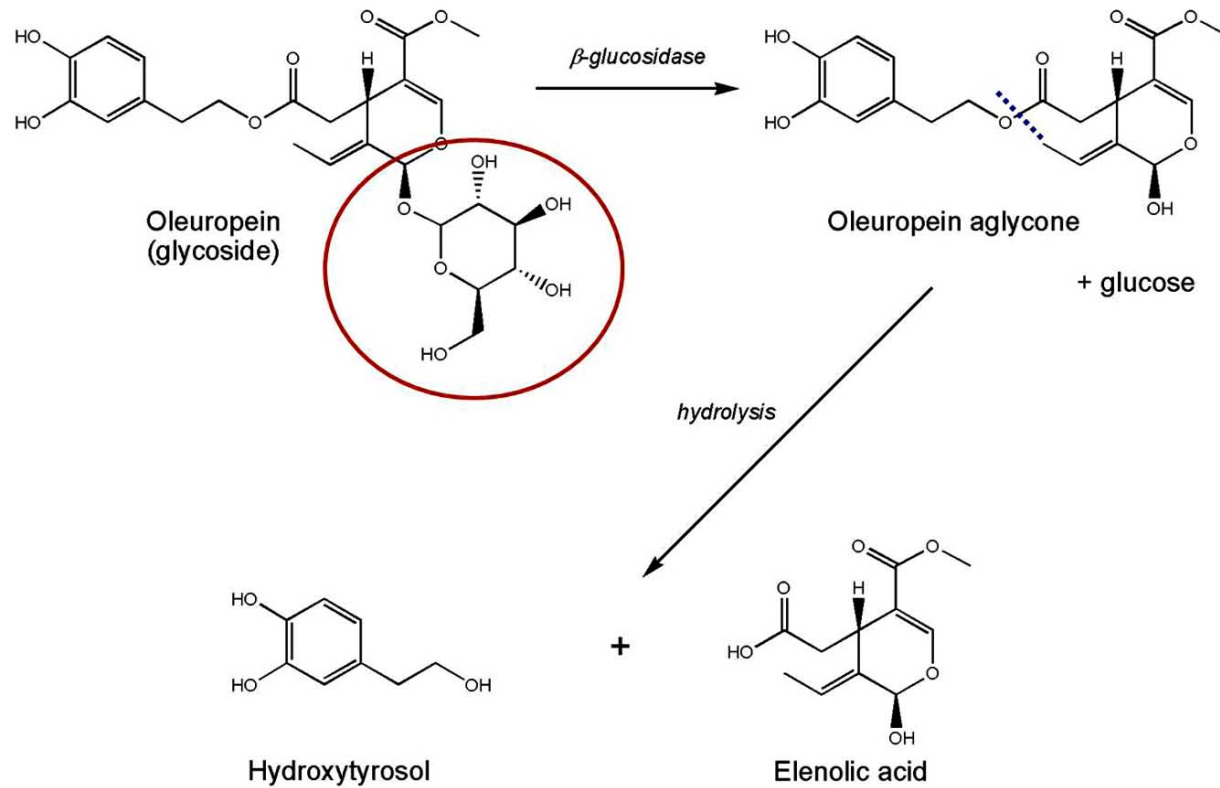

Fig. (3). Metabolism of oleuropein.

\section{A. LC of oleuropein standard}

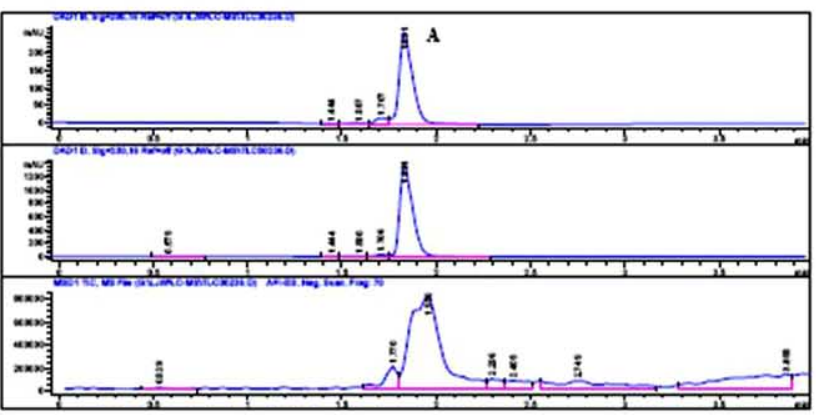

B. MS of oleuropein standard $(\mathrm{M}-\mathrm{H})=\mathbf{5 3 9}$

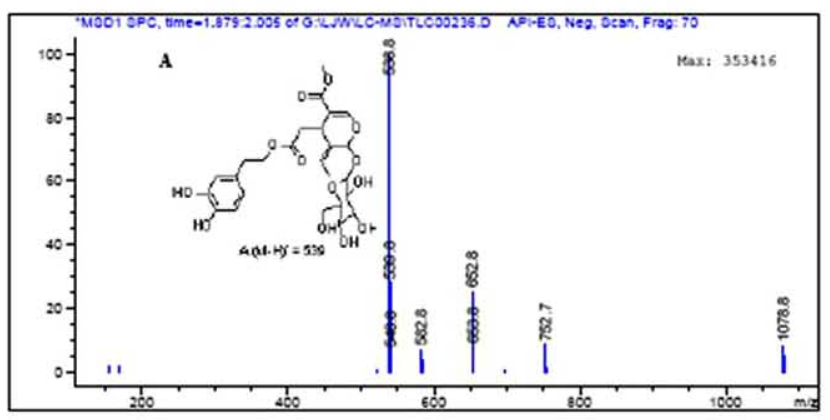

Fig. (4). LC and MS profiles of oleuropein.
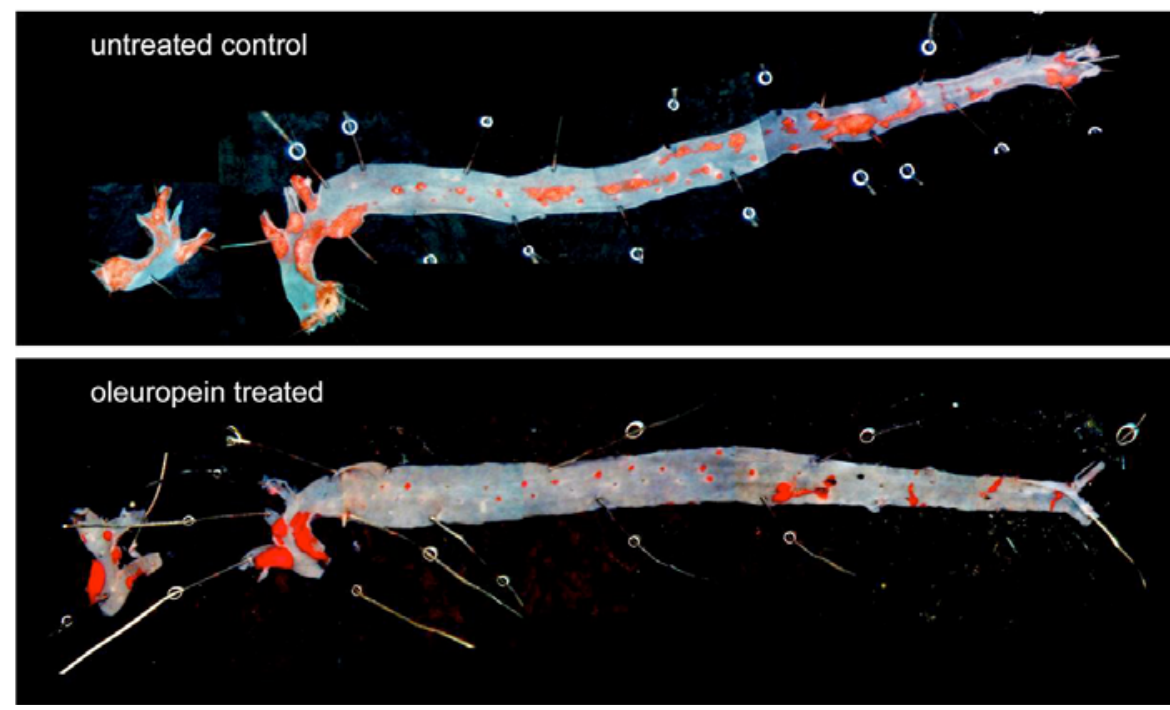

Fig. (5). Oil Red O staining of atherosclerotic plaques in apoE knockout mice.

atherosclerosis by Oil Red O staining Fig. (5) shows representative vessels stained with Oil Red O. The areas of atherosclerotic plaques were quantitated and shown in Fig.
(6). Oleuropein reduced the aortic lesions in both female and male apoE knockout mice. 


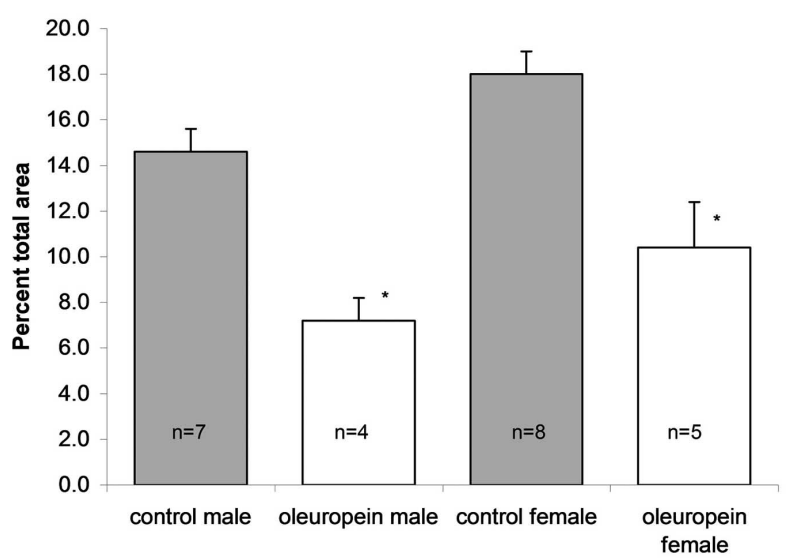

Fig. (6). Quantitation of effect of oleuropein on atherosclerosis in apoE knockout mice.

\section{MOLECULAR MODELING OF OLEUROPEIN BINDING TO PPARS}

We performed detailed theoretical and computational analysis for binding of oleuropein to PPAR $\alpha, \operatorname{PPAR} \gamma$, and PPAR $\delta$, using the known crystal structure of their ligand binding domains. The large ligand-binding site is formed by several $\alpha$-helices. We first used molecular docking to generate several distinct binding orientations, and performed molecular dynamics simulation to further relax the complex. Then, we estimated the affinity for each binding mode, selected the ones with the lowest free energy, and analyzed the detailed interactions.

The known ligand binding domains for PPAR $\alpha$, PPAR $\gamma$, and PPAR $\delta$ are large and Y-shaped. Within these domains, there is variability in how various ligands bind. Long flexible ligands such as the naturally occurring fatty acid eicosapentanoic acid (EPA) can bind in various conformations that are all energetically favorable, suggesting that these alternative conformations are important means of regulation of activity. Our molecular modeling shows that oleuropein binds to the PPARs at least as favorably as known ligands, and its conformations provide insight into possible mechanisms of action. The binding configurations are shown in Figs. (7-9).

Fig. (7a) shows how oleuropein (red and blue) binds to PPAR $\delta$ (yellow backbone). The conformation of oleuropein is compared with those of the fibrate GW2433 (green) and EPA (purple) in Fig. (7b).

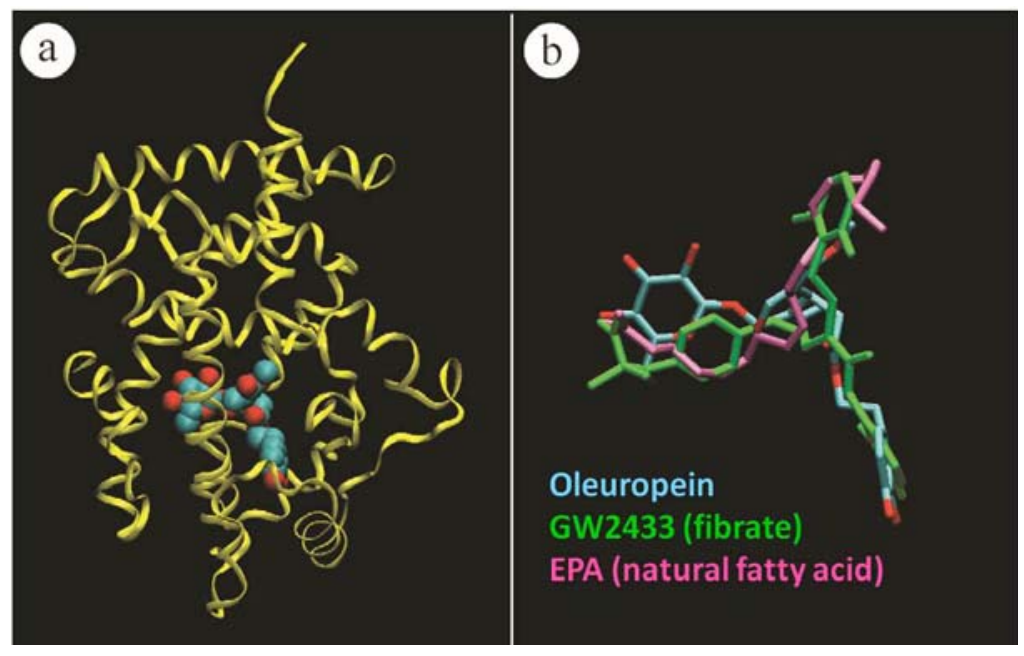

Fig. (7). Binding to PPAR $\delta$.

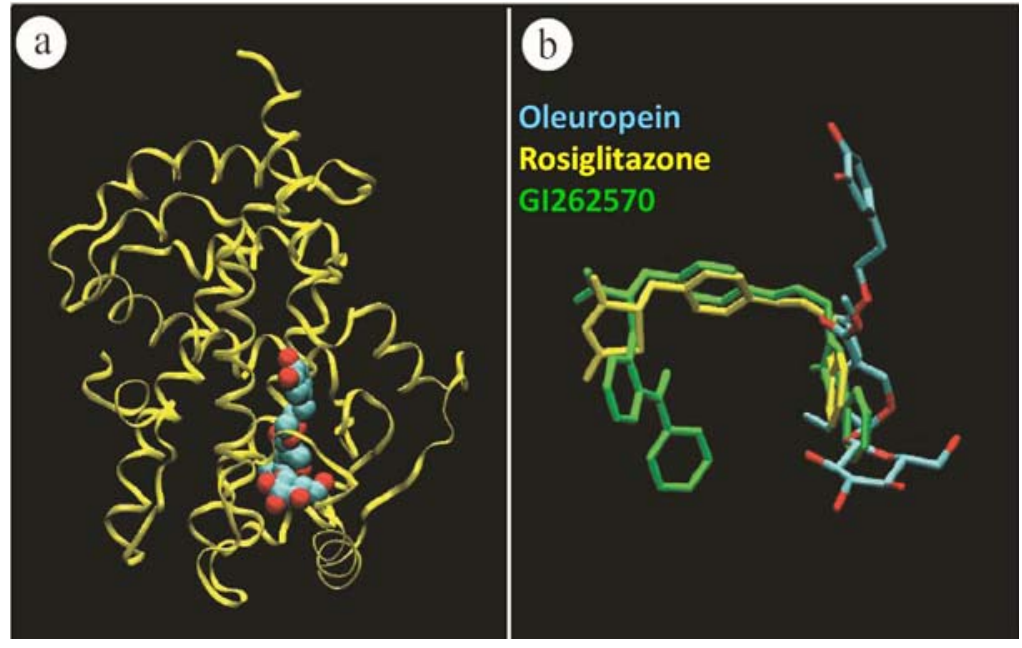

Fig. (8). Binding to PPAR $\gamma$. 


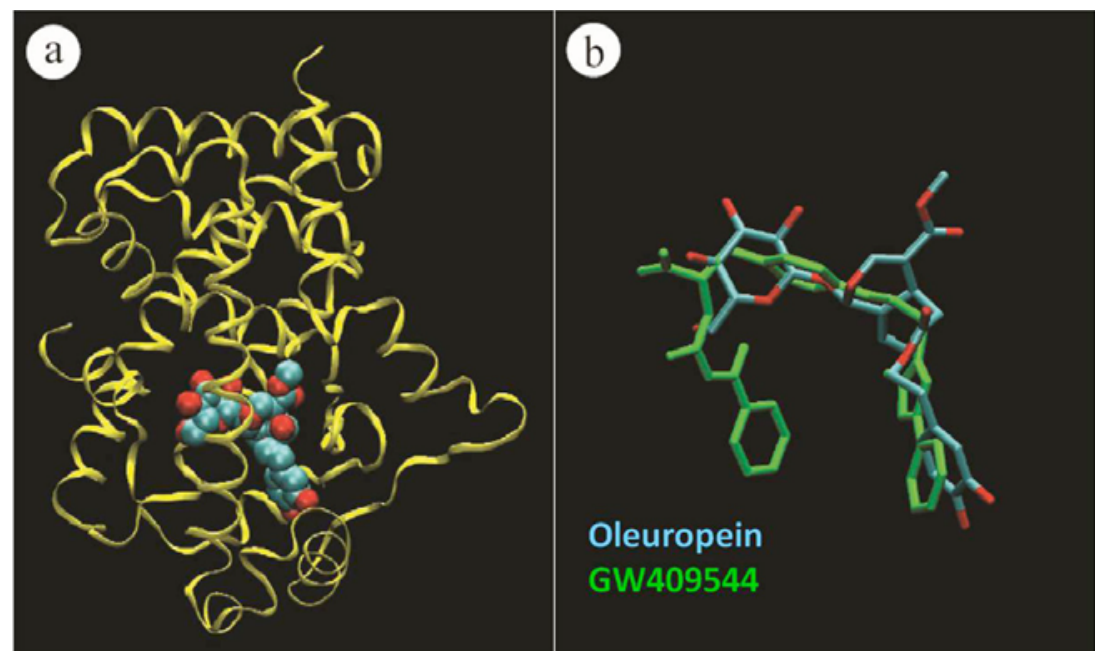

Fig. (9). Binding to PPAR $\alpha$.

Oleuropein mimics precisely the conformations of these known ligands. Fig. (8) shows that binding of oleuropein to PPAR $\gamma$ involves an extended "up-down" configuration, which differs from the U-shaped configuration adopted by the known PPAR $\gamma$ ligands rosiglitazone (yellow) and GI262570 (green). Fig. (9) shows that binding of oleuropein to PPAR $\alpha$ involves a Y-shaped configuration that differs from the U-shaped configuration of the fibrate GW409544 (green). These results suggest that oleuropein binds to the PPARs with distinct conformations that do not necessarily match the conformations of known ligands.

\section{CONCLUSION}

Epidemiologic studies demonstrate that a Mediterranean diet rich in olive oil is associated with decrease in risk for cardiovascular disease, obesity, and diabetes. Although some of the protection is due to unsaturated fatty acids, additional protection may be conferred by small molecules found in olive oil and olive plants, including oleuropein. We report here studies in mice that document the bioavailability of these molecules when administered orally. Using the apoE knockout mouse model fed a high fat diet, orally administered oleuropein significantly reduced the extent of diet-induced atherosclerosis found in the aorta of apoE knockout mice. Molecular modeling studies indicate that oleuropein binds to the PPARs, but with distinct conformations that differ from the way known ligands bind. These results offer a possible explanation for the effects of oleuropein on metabolism and cardiovascular disease.

\section{ABBREVIATIONS}

$\begin{array}{ll}\text { apoE } & =\text { apolipoprotein } \mathrm{E} \\ \mathrm{BMI} & =\text { body mass index } \\ \mathrm{EPA} & =\text { eicosapentanoic acid } \\ \mathrm{HDL} & =\text { high density lipoprotein } \\ \mathrm{LDL} & =\text { low density lipoprotein } \\ \mathrm{LC} & =\text { liquid chromatography } \\ \mathrm{MS} & =\text { mass spectrometry } \\ \text { PPAR } & =\text { peroxisome proliferator activator receptor } \\ \mathrm{TNF} & =\text { tumor necrosis factor }\end{array}$

\section{ACKNOWLEDGEMENTS}

We acknowledge the support of grant R01-AT01383 from the National Center for Complementary and Alternative Medicine (NCCAM), NIH and grant R01AI31343 from the National Institute of Allergy and Infectious Diseases (NIAID), NIH to SLH and grant R01NS33335 from the National Institute of Neurologic Diseases and Stroke (NINDS), NIH to PLH.

\section{REFERENCES}

[1] Willett, W.C.; Manson, J.E.; Stampfer, M.J.; Colditz, G.A.; Rosner, B.; Speizer, F.E.; Hennekens, C.H. Weight, weight change, and coronary heart disease in women. Risk within the 'normal' weight range. JAMA, 1995. 273, 461-5.

[2] Shaper, A.G.; Wannamethee, S.G.; Walker, M. Body weight: implications for the prevention of coronary heart disease, stroke, and diabetes mellitus in a cohort study of middle aged men. BMJ, 1997. 314, 1311-7.

[3] Huang, P.L. A comprehensive definition for metabolic syndrome. Dis. Model. Mech., 2009. 2, 231-7.

[4] Huang, P.L. eNOS, metabolic syndrome and cardiovascular disease. Trends Endocrinol. Metab., 2009. 20, 295-302.

[5] Evans, R.M.; Barish, G.D.; Wang, Y.X. PPARs and the complex journey to obesity. Nat. Med., 2004. 10, 355-61.

[6] Katan, M.B.; Zock, P.L.; Mensink, R.P. Dietary oils, serum lipoproteins, and coronary heart disease. Am. J. Clin. Nutr., 1995. 61, 1368S-1373S.

[7] Tuck, K.L.; Hayball, P.J. Major phenolic compounds in olive oil: metabolism and health effects. J. Nutr. Biochem., 2002. 13, 636644.

[8] Visioli, F.; Poli, A.; Gall, C. Antioxidant and other biological activities of phenols from olives and olive oil. Med. Res. Rev., 2002. 22, 65-75.

[9] Visioli, F.; Galli, C. Antiatherogenic components of olive oil. Curr. Atheroscler. Rep., 2001. 3, 64-7.

[10] Omar, S.H. Oleuropein in olive and its pharmacological effects Sci. Pharm., 2010. 78, 133-154.

[11] Owen, R.W.; Giacosa, A.; Hull, W.E.; Haubner, R.; Wurtele, G.; Spiegelhalder, B.; Bartsch, H. Olive-oil consumption and health: the possible role of antioxidants. Lancet Oncol., 2000. 1, 107-12.

[12] Visioli, F.; Bellosta, S.; Galli, C. Oleuropein, the bitter principle of olives, enhances nitric oxide production by mouse macrophages. Life Sci., 1998. 62, 541-6.

[13] Lee-Huang, S.; Huang, P.L.; Zhang, D.; Lee, J.W.; Bao, J.; Sun, Y.; Chang, Y.T.; Zhang, J.; Huang, P.L. Discovery of smallmolecule HIV-1 fusion and integrase inhibitors oleuropein and hydroxytyrosol: part II. integrase inhibition. Biochem. Biophys. Res. Commun., 2007. 354, 879-84. 
[14] Lee-Huang, S.; Huang, P.L.; Zhang, D.; Lee, J.W.; Bao, J.; Sun, Y.; Chang, Y.T.; Zhang, J.; Huang, P.L. Discovery of smallmolecule HIV-1 fusion and integrase inhibitors oleuropein and hydroxytyrosol: Part I. fusion inhibition. Biochem. Biophys. Res. Commun., 2007. 354, 872-8.
[15] Plump, A.S.; Breslow, J.L. Apolipoprotein E and the apolipoprotein E-deficient mouse. Аnпи. Rev. Nutr., 1995. 15, 495518.

[16] Nakashima, Y.; Plump, A.S.; Raines, E.W.; Breslow, J.L.; Ross, R. ApoE-deficient mice develop lesions of all phases of atherosclerosis throughout the arterial tree. Arterioscler. Thromb., 1994. $14,133-40$.

(c) Huang et al.; Licensee Bentham Open.

This is an open access article licensed under the terms of the Creative Commons Attribution Non-Commercial License (http://creativecommons.org/licenses/by-nc/3.0/) which permits unrestricted, non-commercial use, distribution and reproduction in any medium, provided the work is properly cited. 